\title{
ULTRAVIOLET PHOTOMETRY OF STARS OBTAINED WITH THE CELESCOPE EXPERIMENT IN THE ORBITING ASTRONOMICAL OBSERVATORY
}

\author{
ROBERT J. DAVIS \\ Smithsonian Astrophysical Observatory, Cambridge, Mass., U.S.A.
}

\begin{abstract}
We have used the television photometers in the Celescope OAO experiment to measure the far ultraviolet brightnesses of several thousand stars, including parts of the constellations Draco, Lyra, Puppis, Vela, Taurus, and Orion; and the Moon. As of this date (22 July 1969), three of our four cameras continue to operate satisfactorily, and we are making measurements in additional star fields distributed throughout the sky. Our shortest wavelength band, which includes the Lyman $\alpha$ line of atomic hydrogen, provides information on the Earth's outer atmosphere, as well as on star brightnesses. The intensity of the Lyman $\alpha$ radiation from the geocorona is a maximum when the Sun is near the horizon as seen by the OAO, and a minimum when the Sun is in the nadir. The direction that the telescope points does not much affect the measured intensities.

Because of the heavy logistic problems of identification, calibration, and measurement for so many stars and because of the survey character of the program, the scientific interpretation of the results is, as expected, lagging the measurement program. However, one consistent picture beginning to emerge from our data is that our observed stars are about equally divided between those that fall within 0.5 magnitude of the predicted ultraviolet brightnesses and those that are significantly fainter than predicted. Most of the giant stars we observe exhibit these ultraviolet deficiencies. Since some of these giants are stars for which little or no interstellar reddening is predicted, we attribute the observed deficiencies to the stars themselves.

Many of the objects we observe do not have accurate ground-based published data regarding magnitude, color, and spectral type; new ground-based observations of these objects are required to ensure satisfactory interpretation of our results.
\end{abstract}

\section{Introduction}

The Celescope experiment consists of four ultraviolet television photometers, each with an aperture of $31 \mathrm{~cm}$, mounted in the Orbiting Astronomical Observatory (OAO). These cameras produce pictures, $3^{\circ}$ square, in four spectral regions: U1, extending from 2200 to $3200 \AA$; U2, 1600 to $3200 \AA$; U3, 1350 to $2000 \AA$; and U4, 1050 to $2000 \AA$. A complete description of the instrumentation appears in Smithsonian Astrophysical Observatory Special Report No. 282, 18 July 1968.

Since launch on 7 December 1968, the Celescope experiment has taken 3652 pictures, in 1276 different directions, covering approximately $6 \%$ of the sky. Most of these observations are within $20^{\circ}$ of the galactic plane; however, we have taken enough pictures at high galactic latitude to confirm our expectation that very few objects at high latitudes are brighter than our limit of 9th magnitude at spectral type A0. Near the north galactic pole, we detected about 1 star for every 3 sets of pictures $(1$ star per 12 square degrees), whereas near the Orion nebula, we measured 20 stars per picture, and in the galactic plane in Vela, we measured 50.

Each Celescope picture consists of 256 lines, each of which contains 251 picture elements. The Celescope instrumentation encodes the signal amplitude in each ele- 
ment to 7-bit accuracy. Although Celescope is capable of other modes of picture transmission, these modes have not yet been used for the collection of scientific data. These pictures, recorded on magnetic tape at both the data-acquisition station and the central control center, are re-formatted by NASA's data-analysis center and processed on Smithsonian Astrophysical Observatory's CDC-6400 computer to provide identification and ultraviolet magnitudes for the stars observed. A detailed description of these analysis techniques will appear in Smithsonian Astrophysical Observatory Special Report No. 310, scheduled for publication later this year.

We will publish these Celescope observational results in a series of Celescope Observational Data Reports, which will be regularly distributed to all institutions and individual scientists who request them from the Publications Division, Distribution Section, Smithsonian Astrophysical Observatory, Cambridge, Mass. 02138. Our preliminary review of the pictures and of the resultant data indicates that for nearly every object we have observed, the ultraviolet brightness can be reasonably explained on the basis of atmospheric theory and interstellar reddening. We have, therefore, concentrated our attention on compiling and refining our observational results, postponing the scientific interpretation to a later date. The remainder of this paper is an example of one set of Celescope pictures, typical of the more than one thousand such sets obtained to date.

\section{Sample Celescope Observational Data Report}

Celescope observations are obtained only by live video transmission from the OAO to one of five ground stations: Madagascar (M); Orroral, Australia (O); Quito, Ecuador (Q); Rosman, North Carolina (R); and Santiago, Chile (S). Such transmission can occur only during a 'contact' or 'pass' of the satellite over the station. Contacts are identified by a letter designating the station and a number indicating the number of times the satellite has crossed the ascending node of its orbit. During a contact, the Celescope experiment may transmit video data of one or more exposures. The first of these exposures may precede arrival of the OAO over the station.

The reports are divided into sections, one for each contact. The first page of each section is a 'data sheet' listing the conditions under which the exposures were made. If there are more than three exposures, subsequent exposures are listed on a second page. On this data sheet, right ascension and declination are given for the Celescope optic axis in current-epoch coordinates. Magnetic coordinates are based on an approximate magnetic coordinate system whose north pole is at geographic latitude $78^{\circ} .3 \mathrm{~N}$ and longitude $69^{\circ} .0 \mathrm{~W}$; magnetic hour angle is measured eastward from the sun; beta angle is the distance of the target from the Sun. Roll (direction to the north celestial pole) is measured clockwise from the left end of the filter split line. The directions to zenith, magnetic pole, and Sun are measured counterclockwise from the left end of the filter split line, as indicated by the horizontal lines in the plots and pictures.

The most important parameters of Celescope operation are exposure time and 
operating mode, which control sensitivity and noise level, respectively. Normally, the exposure time is set at $60 \mathrm{sec}$ and the operating mode is set at pulse code modulation (PCM). Blank entries indicate which, if any, cameras were not used.

Status at estimated time of arrival (ETA) lists the less important parameters of operation. Special Report 310 describes these entries in full.

Following the data sheet for each contact are charts, pictures, and numerical data for each exposure. The picture presents the video data exactly as recorded on the magnetic data tape, free from any processing other than that necessary to convert the magnetic data into printed picture. It can thus be used both to assess image quality and to serve as orientation while the numerical data are studied. The small plot, to the same scale as the picture, indicates rough brightnesses and positions for all stars found in the picture and the position of the filter split line. Star-like objects evident in the picture but missing from the small plot include noise pulses, the signal from the calibrator lamp, permanent target blemishes, and stars too near the edge of the field to allow proper analysis of the data. These objects are indicated on the large plot, as described in the paragraph below.

The large plot identifies the star-like images in the picture and cross references them to the data listing. Numbers refer to stars; letters refer to other star-like objects in the picture: $C$ for calibrator lamp; $G$ for ghosts of the calibrator lamp; $F$ for target blemishes ('false stars'); $\mathrm{N}$ for noise pulses; and $\mathrm{X}$ for objects more than 1.4 from the optical axis. Asterisks refer to merged images for double stars that are seen as separate objects on some, but not all, cameras. These reports list numerical data only for the numbered objects and for those represented by asterisks.

Ultraviolet brightnesses and identifications are presented in the observational data listing following the pictures and identification charts for each exposure. Objects are listed and assigned identification numbers in order of increasing right ascension. For each object, the first line summarizes ground-based data, and subsequent lines list the Celescope observational data, one line per camera. If the Celescope identification catalog contains more than 10 literature references for an object, a second line is used for printing additional reference numbers.

The information is listed as follows:

ID Identification number.

HD Henry Draper Catalogue number, or variable star name.

DM Durchmusterung number or IC/NGC number.

R.A. Right ascension (1950.0).

DEC. Declination (1950. 0).

MAGNITUDES Magnitudes (M1, M2, M3), and magnitude code describing same. AND MCODE The preferred set of magnitudes $(V, B-V, U-B)$ is assigned a magnitude code of 1 . Refer to CDL-100 for other code assignments.

SP

$\mathrm{L}$ Spectral type.

Luminosity class. 


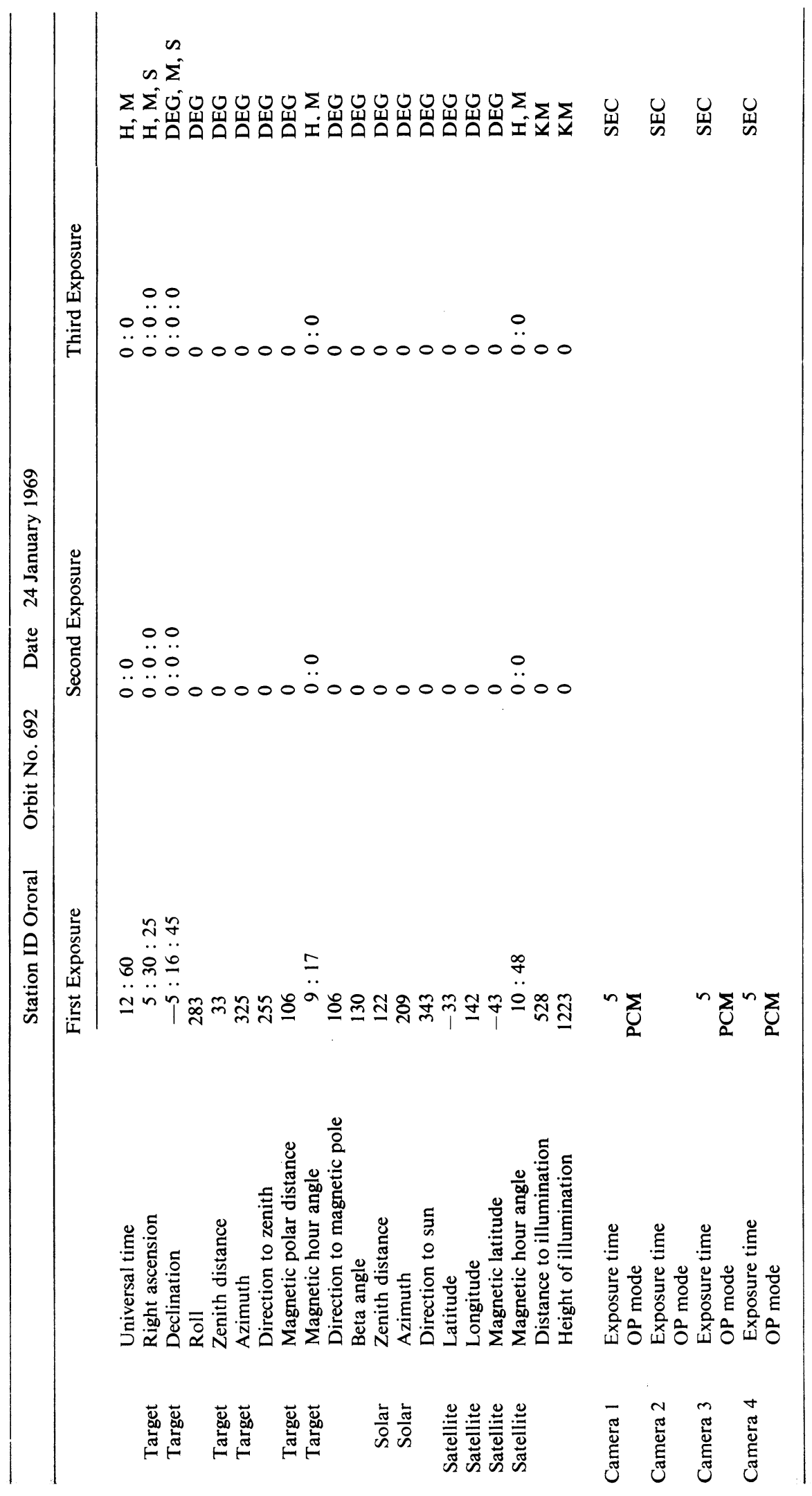




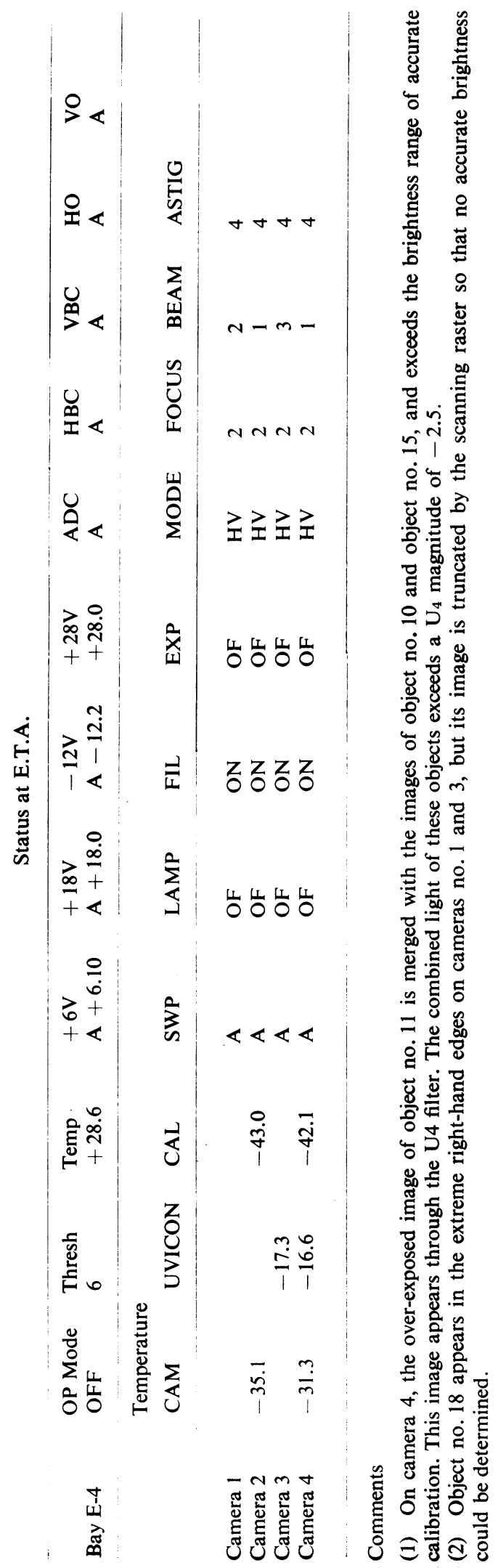


RIGHT ASCENSION: $05^{\mathrm{h}} 30^{\mathrm{m}} 25^{\mathrm{s}}$

DECLINATION : $-05^{\circ} 16^{\circ} 45^{\prime \prime}$
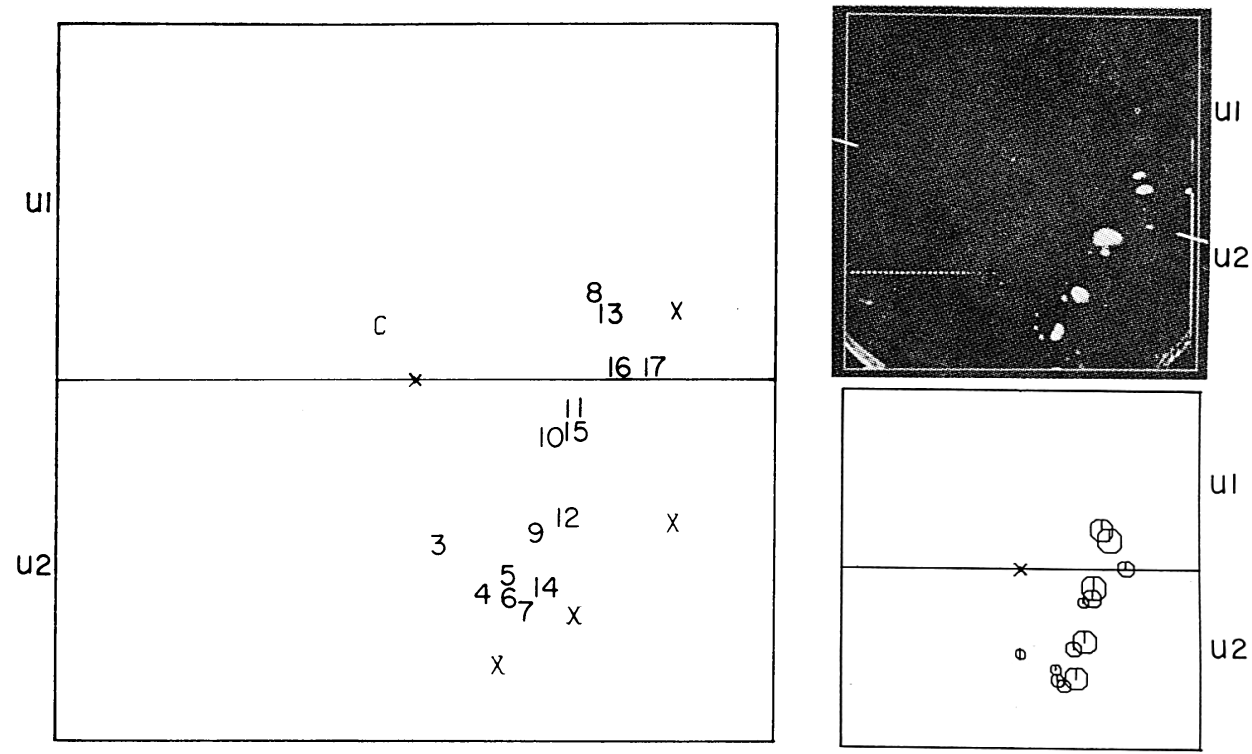

A097- 3 . 0692

CAMERA 1

A097- 3 . 0692
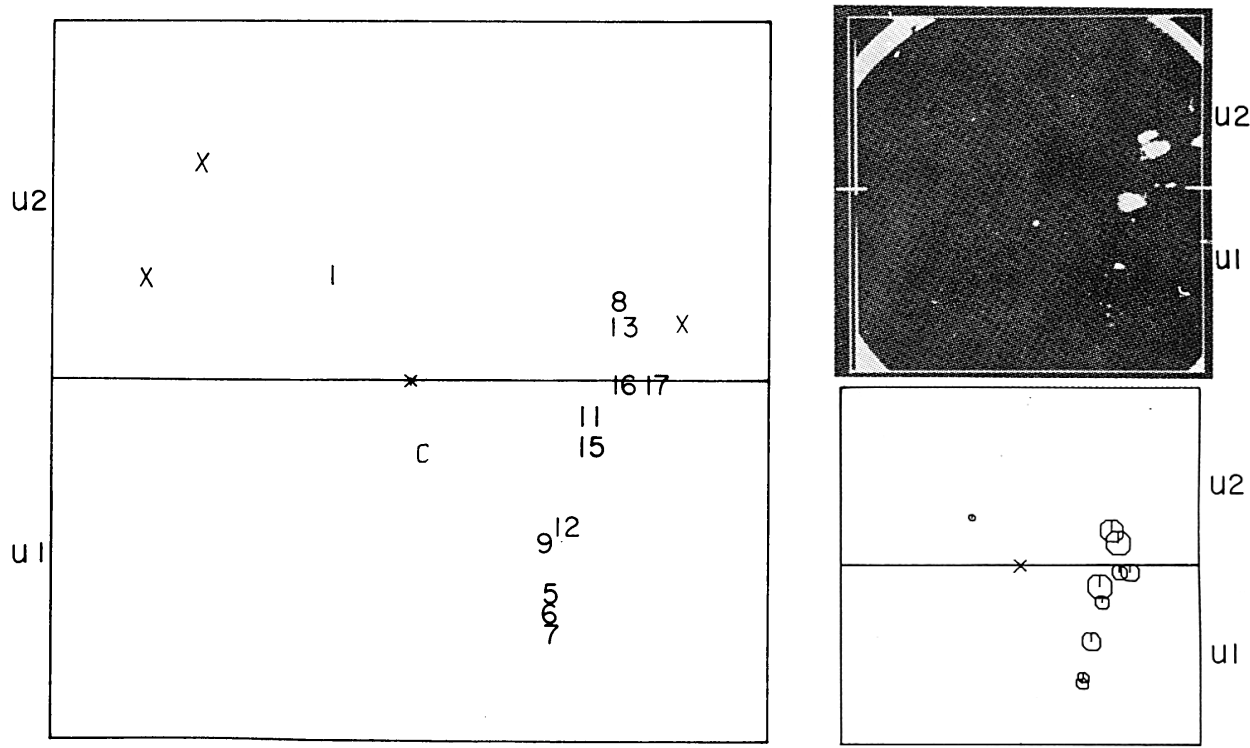

A097- 4,0692

CAMERA 3

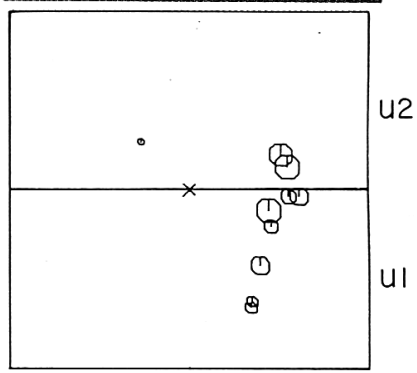

A097- 4 . 0692

Fig. 1. 


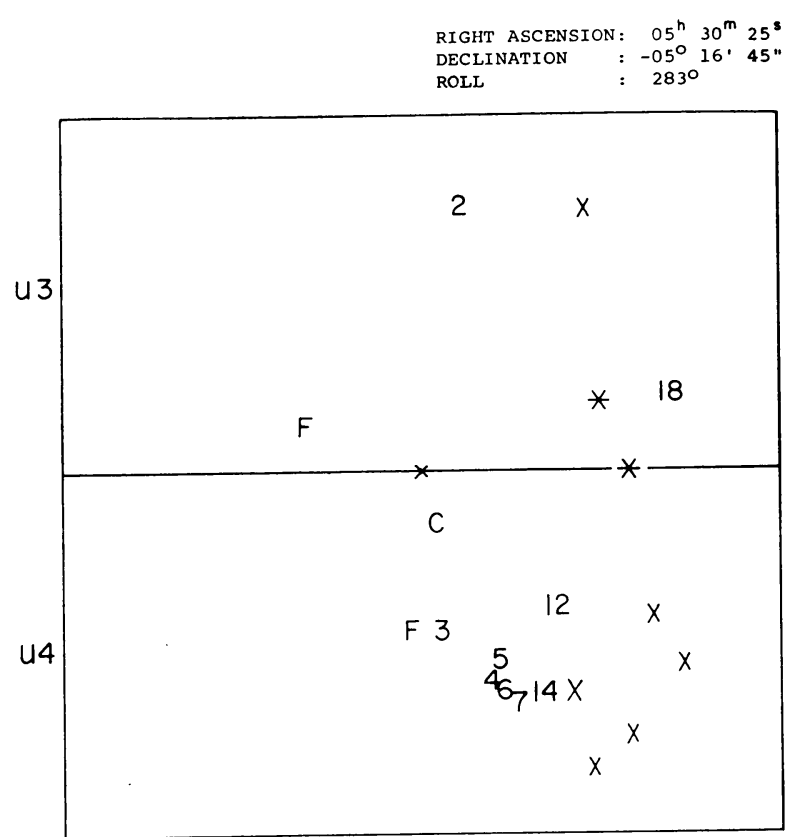

A097- 5 . 0692
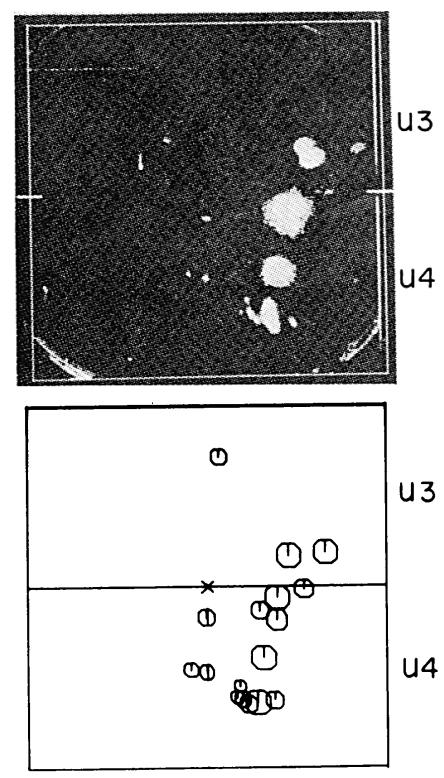

A097- 5. 0692

Fig. 2. 


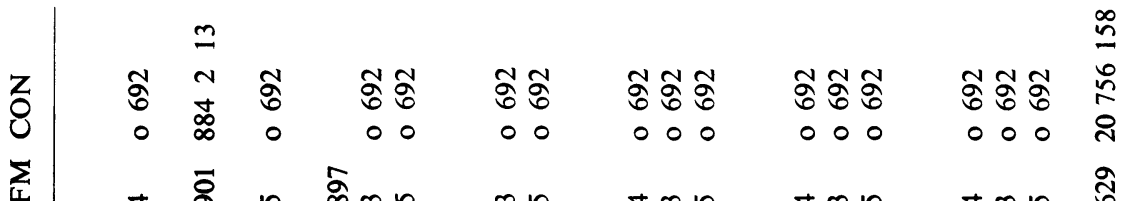

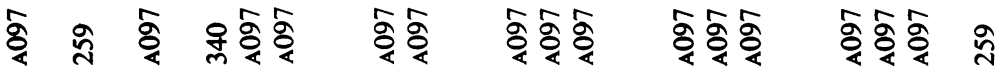

¿

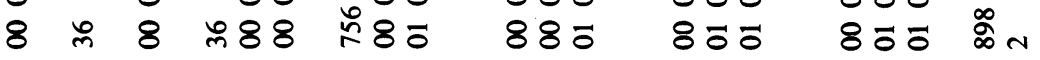
$m$ กิ

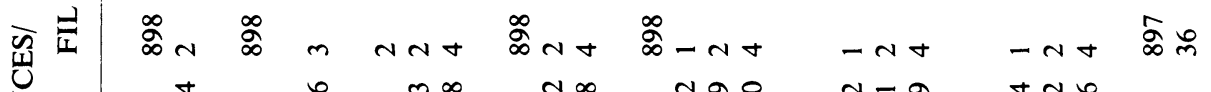

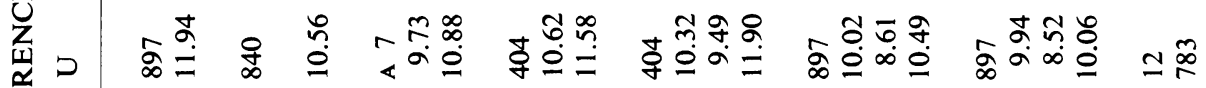

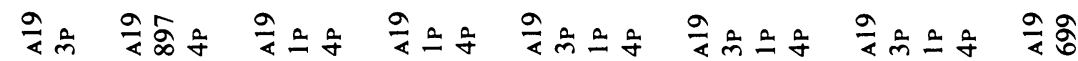

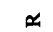

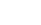

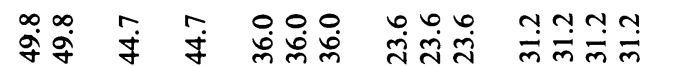

in

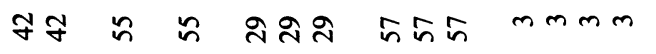

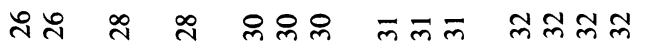
nin $n$ in num oิ

ก)

$\stackrel{n}{n}$

8
$\infty$
0

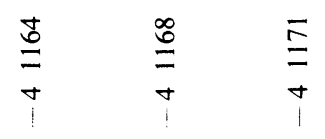

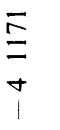

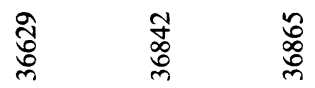

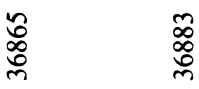

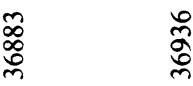

๓̊

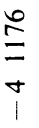

$\frac{2}{m}$ 
ช

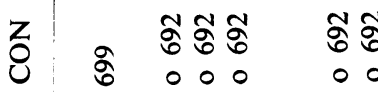

$\sum_{L} \stackrel{\infty}{=} m+n \quad \forall m$

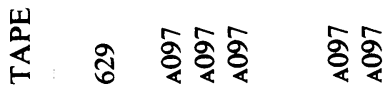

藏

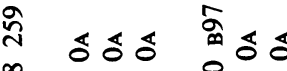

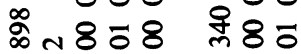

$\sum_{\mathrm{Z}}$

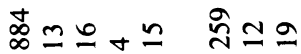

离茫

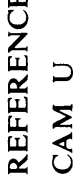

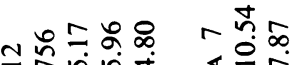

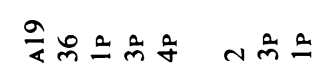

$\propto$

$\begin{array}{ll}\infty & 0 \\ \vdots & \\ \vdots & >\end{array}$<smiles>CC1CCCCCC1</smiles>

足

2

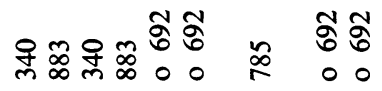

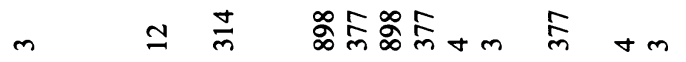

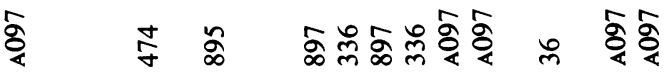

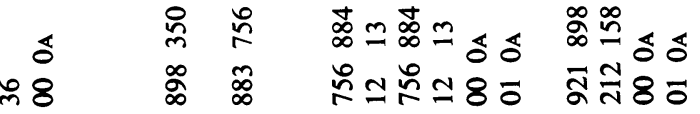

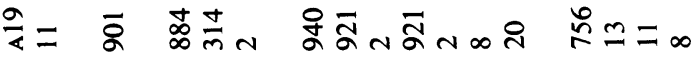

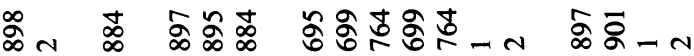

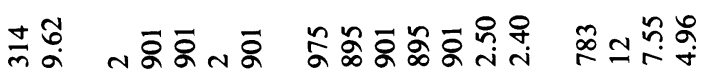

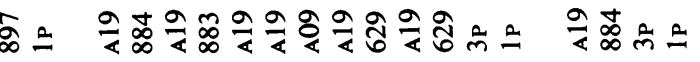

के ठे 原

$\stackrel{?}{i}$

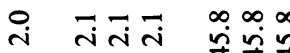

岀

1 1

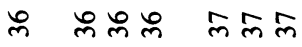

< స సెల్లెల్లిల్లు

$\propto$

$n$ nnn $n n n$

$\sum$

응ำ
岕

$\stackrel{2}{\underline{\Sigma}}$

i

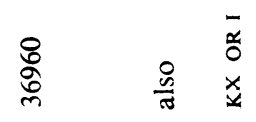

ara

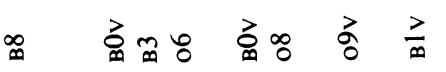

$--6---6--$

के $\infty$ क त

$\begin{array}{llllll}1 & 1 & 1 & 1 & 1 & 1\end{array}$

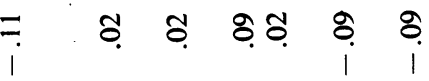

๙

芯

$\tilde{\sigma}$

ণุ

ริ

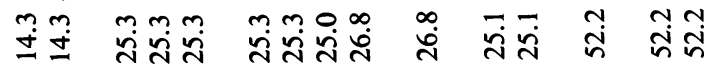

po nun pon p pu

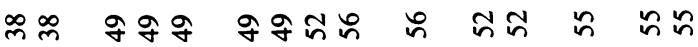

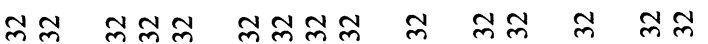

nin nun nunn $n$ in $n$ in

$\overline{\bar{m}} \stackrel{m}{m} \frac{n}{m} \stackrel{n}{m} \bar{m} \frac{a}{m} \quad \stackrel{\infty}{=}$

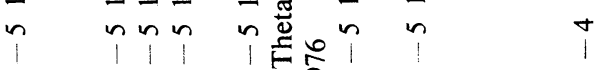

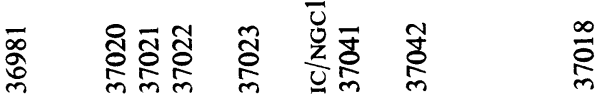

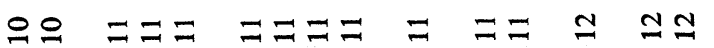


Nลิ

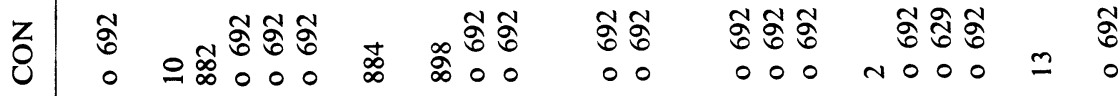

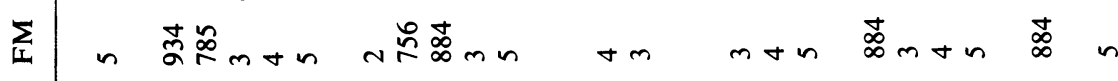

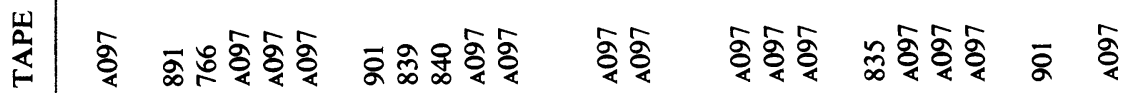

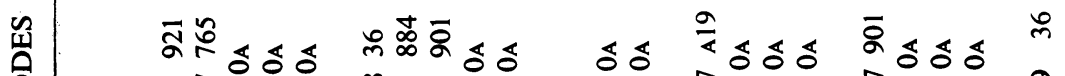

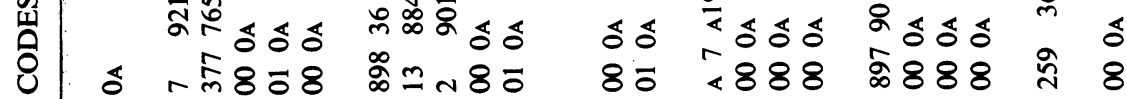
$\sum$ 年

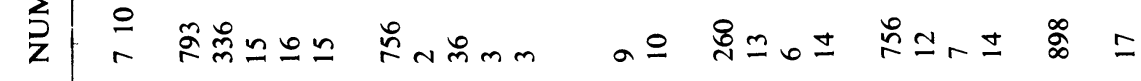

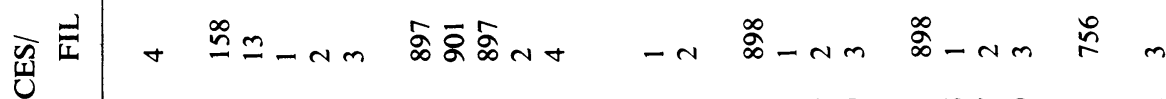

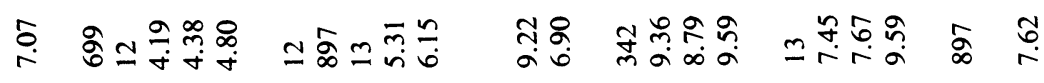

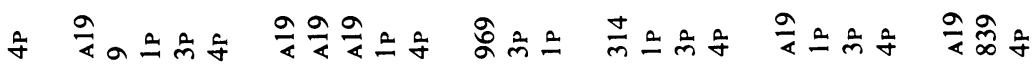

$\propto \propto$ 
$6 \ldots 7 \ldots 8$

REFERENCES/ References to original publications of the data. CDL-100 contains the bibliography. References are listed in the same columns used to report the Celescope observational results.

CAM

$\mathrm{U}$

FIL

NUM

CODES

TAPE, FM

CON

Special characteristics and peculiarities. Refer to CDL-100 for code assignments.

Camera number and operation mode; P is for direct PCM mode, A for analog mode, and $\mathrm{S}$ for store mode.

Ultraviolet magnitude: $U=-2.5 \log I$, where $I$ is the measured intensity in MKS units (watts $\mathrm{m}^{-3}$ ).

Filter number.

An identification number by which the computation for each object can be traced and verified.

Five columns describing the quality of the data. The first column describes the background in the vicinity of the object: $\operatorname{codes} 0$ and 2 are normal background; 1 and 3 indicate unreliable data because of high background surrounding the object.

The second column describes the relationship of the object to the filter split line; codes greater than or equal to 2 indicate unreliable data because of proximity to the filter split line.

The third and fourth columns indicate general picture quality, larger numbers indicating poorer quality. The fifth column is a letter indicating which calibration table was used for data reduction. Special Report 310 includes a complete description of these quality codes.

Tape number and frame number by which the supporting data can be located in the Celescope files.

Contact designation.

\section{Discussion}

Henize: I understand that you find the excess UV radiation of $\theta$ Ori to originate, at least partially, in the Orion nebula. Could you say whether your observation of $\eta$ Car relates to the star itself or to its surrounding nebula?

Davis: The observation of $\eta$ Car does not have sufficient resolution to determine definitely whether the observed UV comes only from the star, or partly from the nebula. However, I believe it is best interpreted as including an important contribution from the nebula.

Morton: Does the Orion nebula appear bright in all your wavelength bands?

Davis: Yes.

Malaise: When will Celescope data be released and how will it be made available?

Davis: Celescope data will be released in regular reports (Celescope Observational Data Reports), beginning in about two months. Any scientist wishing to receive these reports should write to the Publications Division, Distribution Section, Smithsonian Astrophysical Observatory, Cambridge, Mass. 02138, USA, requesting that he be placed on the mailing list. 